\title{
Pacientes com Crises Epilépticas e Lesões Expansivas Cerebrais Submetidos à Exérese Restrita da Lesão ou Associada à Área Irritativa Adjacente *
}

\author{
Aziz Rassi Neto
}

Pacientes com tumores cerebrais podem apresentar crises epilépticas, e muitas vezes refratárias ao tratamento clínico, como único sintoma da presença dessas lesões. Por esta razão é necessária uma investigação criteriosa, onde a tomografia computadorizada craniana e ressonância magnética cerebral são métodos ideais para surpreender processos expansivos como fator etiológico das crises epilépticas. Este estudo compreende 37 pacientes com crises epilépticas e lesões expansivas cerebrais, provenientes do Departamento de Neurologia e Neurocirurgia da Universidade Federal de São Paulo - Escola Paulista de Medicina. Após a seleção destes pacientes, todos foram submetidos à intervenção neurocirúrgica e eletrocorticografia intra-operatória. Nosso objetivo é analisar qual o melhor procedimento a ser adotado em pacientes com crises epilépticas e lesões expansivas cerebrais, isto é, a exérese cirúrgica restrita à lesão expansiva ou associada à da área irritativa (demonstrado pela eletrocorticografia), baseando-nos na evolução clínica em relação às crises epilépticas e aos resultados eletrencefalográficos e eletrocorticográficos.

Nossa casuística foi composta de 21 pacientes do sexo masculino e 16 do sexo feminino. A faixa etária variou entre 9 e 66 anos.

O material foi dividido em dois grupos: Grupo 1 - Formado por 21 pacientes com crises epilépticas "não controladas", fazendo uso de medicamentos antiepilépticos em doses adequadas e que apresentavam uma ou mais crises parciais por semana ou uma ou mais crises generalizadas por mês. Grupo 2 - Composto de 16 pacientes com crises epilépticas "controladas" fazendo uso de medicamentos antiepilépticos em doses adequadas e que não mais apresentavam crises.
No material descrito analisamos:

I. Freqüência das crises epilépticas após a cirurgia em relação aos procedimentos cirúrgicos adotados nos 37 pacientes.

II. Análise dos eletrencefalogramas e eletrocorticogramas em relação à presença das descargas paroxísticas focais nos pré e pós-operatórios.

I - Dos 21 pacientes (grupo 1) com crises "não controladas", onze foram submetidos à exérese da lesão e da zona epileptógena adjacente (até $1,5 \mathrm{~cm}$ da margem da lesão cerebral) demonstrada pela ECoG (subgrupo A). Em dez (de 21) a exérese ficou restrita ao processo expansivo (subgrupo B), sendo que em cinco casos os traçados mostraram-se sem atividade irritativa após a exérese da lesão, e em cinco ainda havia atividade irritativa em áreas consideradas eloquientes que, se lesadas, poderiam levar a sequielas neurológicas.

Nos 16 pacientes com "crises controladas" (grupo 2) a eletrocorticografia também foi feita antes e após a exérese da lesão. Nestes casos, mesmo havendo uma área com atividade irritativa focal persistente, adjacente à lesão expansiva cerebral, ela não foi retirada. Dos 11 pacientes (de 21) submetidos à exérese da lesão associada à área irritativa (subgrupo A), $10(91 \%)$ evoluíram sem crises epilépticas. Dos dez (de 21) submetidos à exérese restrita da lesão (subgrupo B), quatro (40\%) persistiram com crises, porém

\footnotetext{
Tese apresentada à Universidade Federal de Sāo Paulo - Escola Paulista de Medicina - para obtenção do titulo de Doutor em Medicina. Orientador: Prof. Dr. Fernando A. Patriani Ferraz, São Paulo, 1995
} 
com menor frequiência em relação ao pré-operatório, e seis $(60 \%)$ evoluíram sem crises. Dentre os 21 pacientes do grupo $1,76,25 \%$ evoluíram sem crises, tendo o mesmo ocorrido em $100 \%$ dos casos do grupo 2 (16 casos); dos 37 pacientes operados $86,5 \%$ evoluíram sem crises.

II - Comparando os resultados dos EEGs antes e após a cirurgia no grupo 1 ( 21 casos), foi observada importante queda percentual dos traçados com descargas paroxísticas focais - de 71,5\% (pré-operatório) para $28,5 \%$ (pós-operatório). Dos 11 pacientes que foram submetidos à exérese da lesão associada à área irritativa, três persistiram com descargas paroxísticas, e dos dez (de 21) que foram submetidos à exérese restrita da lesão, três persistiram com estas descargas. No grupo 2, foi observada uma queda desta atividade de $31 \%$ para $6 \%$ no pós-operatório. Na análise geral dos EEGs ( 37 casos) foi observada uma queda estatisticamente significante dos traçados com descargas paroxísticas de $54 \%$ para $19 \%$, no pós-operatório. O estudo comparativo entre os grupos 1 e 2 demonstrou que o número de EEGs com descargas paroxísticas (no pré-operatório) foi significantemente maior no grupo 1 do que no grupo 2 .

Nos eletrocorticogramas do grupo com crises "não controladas" ( 21 casos), foi observada uma grande queda percentual de traçados com atividades paroxísticas $90,5 \%$ para $38 \%$, no pós-operatório. Dos 11 pacientes submetidos à exérese da lesão associada à área irritativa, três $(27 \%)$ persistiram com descargas paroxísticas após a cirurgia, e dos dez submetidos à exérese restrita da lesão, cinco (50\%) persistiram com estas descargas. No grupo 2 foi observada uma queda dos traçados com descargas paroxísticas, de $60 \%$ para $27 \%$.

A análise geral dos ECoGs ( 37 casos) demonstrou uma redução importante dos traçados com descargas paroxísticas após a cirurgia, isto é, de $78 \%$ para $32,5 \%$. O estudo comparativo entre os grupos 1 e 2 demonstrou que o número de ECoGs com descargas paroxísticas (pré-exérese da lesão) foi significantemente maior no grupo 1 do que no grupo 2 . $\mathrm{O}$ número de traçados com descargas paroxísticas foi maior nos eletrocorticogramas $(78 \%)$ do que nos eletrencefalogramas $(54 \%)$, no pré-operatório.

\title{
TESE
}

\section{Ectasia da Artéria Basilar e Acidente Vascular Cerebral: Estudo de 21 Casos *}

\author{
Roberto de Magalhães Carneiro de Oliveira
}

A ectasia da artéria basilar (EB) é a dilatação do calibre em toda ou em parte de sua extensão, e/ou quando for anormalmente tortuosa em seu trajeto. Associa-se freqüentemente ao seu alongamento. É entidade clínica e patológica rara. Pode estar presente desde o nascimento, sendo assintomática. As manifestações clínicas são decorrentes de compressão de nervos cranianos, fenômeno isquêmico ou hemorrágico, síndrome de pseudotumor ou hidrocefalia. Procuramos descrever casos da associação de EB e AVC, analisar sua freqüência, aspectos clínicos e radiológicos, e os mecanismos envolvidos nas diferentes formas de apresentação clínica desta entidade. Encontramos, entre 3.250 pacientes com história de doença cerebrovascular, $21 \mathrm{com}$ AVC e EB. A associação de EB e AVC foi prevalente em indivíduos do sexo masculino após
50 anos. Os principais sintomas observados foram hemiparesia, alteração de nervos cranianos e ataxia cerebelar. Na instalação do AVC foram importantes as tonturas rotatórias e a cefaléia. Os infartos relacionados à EB puderam ser atribuídos a diferentes mecanismos: trombose da artéria, embolia artério-arterial, efeito de massa com angulação e obstrução de ramos das artérias vertebrais e basilar. A ressonância magnética mostrou-se o exame de diagnóstico por imagem mais completo para avaliação das relaçōes entre a $\mathrm{EB}$ e as estruturas adjacentes.

\footnotetext{
Tese apresentada à Escola Paulista de Medicina para a obtenção do título de Mestre em Neurologia. Orientador. Prof. Dr. José Geraldo C. Lima. Coorientador: Prof. Dr. José Osmar Cardeal, Sāo Paulo, 1994
} 


\title{
Potencial Evocado Somatossensitivo por Estimulação do Nervo Cutâneo Lateral da Coxa. Contribuição ao Estudo Eletrofisiológico da Meralgia Parestésica *
}

\author{
Roseli Aparecida Freire
}

Esta pesquisa incluiu a análise do potencial evocado somatossensitivo por estimulação do nervo cutâneo lateral da coxa. Foi também avaliada sua utilização em pacientes com Meralgia Parestésica. O estudo abrangeu 3 fases, sendo que na fase I foi definido que a intensidade de estímulo a partir de 3 limiares sensitivos foi suficiente para obter-se a menor latência e a maior amplitude do primeiro componente positivo da resposta evocada (P1). Na fase II, foram examinados 80 nervos e foi determinado que o Limite $\mathrm{Su}$ perior de Confiança é de 33,8 ms (mediana de 29,6 ms) para a latência e o Limite Inferior de Confiança é de 0,29 $\mathrm{uV}$ (mediana de $0,73 \mathrm{uV}$ ) para a amplitude do componente $\mathrm{P} 1$. Na fase III foi avaliado um grupo de pacientes com o diagnóstico de Meralgia Parestésica e os resultados permitiram afirmar que houve diferença significante para os valores de latência e amplitude do P1 entre os pacientes e os indivíduos sadios. Concluiu-se que o potencial evocado somatossensitivo por estimulação do nervo cutâneo lateral da coxa pode dar uma importante contribuição na avaliação neurofisiológica deste nervo.

Tese apresentada à Escola Paulista de Medicina para obtenção do título de Mestre pelo curso de Pós-Graduação em Neurologia. Orientador: Prof. Dr. Joăo Antonio Maciel Nóbrega, Săo Paulo, 1991

\section{TESE}

\section{Crises Epilépticas após Acidente Vascular Cerebral por Infarto Cerebral: Estudo de 35 Pacientes*}

\section{Márcia Maiumi Fukujima}

Acidente vascular cerebral (AVC) constitui a $3^{\text {a }}$ causa de morte no mundo e estima-se que somente $30 \%$ dos pacientes recuperem todas as funções. As principais seqüelas são motora e de linguagem, porém crises epilépticas ocorrem em cerca de $6 \%$ dos casos. Considerando-se a relevância da ocorrência de crises epilépticas após o acidente vascular cerebral isquêmico (AVCI) por infarto cerebral, estudamos um grupo de 35 pacientes com AVCI que evoluíram com crises epilépticas (Grupo 1) coimparando-o a um grupo de 35 pacientes controle com $\mathrm{AVCl}$ que evoluíram sem crises epilépticas (Grupo 2), com o objetivo de identificarmos fatores clínicos e laboratoriais que possam ser preditivos para ocorrência de crises epilépticas após AVCI, e analisar as principais características dessas crises epilépticas. Idade, sexo, raça e antecedentes de hipertensão arterial, diabetes melito, enxaqueca, cardiopatia e doen- ças vasculares cerebrais prévias não constituíram fatores preditivos para crises epilépticas após AVCI, assim como valores de hematócrito, glicemia, colesterol total e frações HDL e LDL, triglicérides, anormalidades eletrocardiográficas e liquóricas. Houve maior freqüência de tabagistas e etilistas no Grupo 1 do que no Grupo 2. Lesões extensas relacionaram-se ao Grupo 1 As crises epilépticas foram na maioria dos casos parciais e iniciaram-se após a fase aguda do AVCI; sua recorrência foi pouco freqüente e o controle medicamentoso foi facilmente obtido.

\footnotetext{
Tese apresentada à Escola Paulista de Medicina para obtenção de Título de Mestre em Neurologia. São Paulo - 1994. Orientador: Prof. Dr. José Geraldo de Camargo Lima. Co-Orientador: Prof. Dr. José Osmar Cardeal
} 


\title{
- TESE
}

\section{Miopatia Centronuclear: Estudo de dez pacientes}

\author{
Edmar Zanoteli
}

Foram apresentados, neste estudo, dez pacientes com miopatia centronuclear, com o objetivo de analisar seus aspectos clínicos e laboratoriais. Os pacientes foram avaliados através de história clínica, de exames clínico e neurológico, de exames subsidiários e biopsia muscular. Houve uma predominância no sexo feminino e na cor branca. Sete pacientes foram esporádicos e herança autossômica recessiva foi sugerida em dois. Manifestações gestacionais e neonatais foram freqüentes, incluindo-se diminuição na movimentação fetal, polihidrâmnio materno, hipóxia perinatal, hipotonia muscular, choro e sucção fracos, e disfagia. Atraso no desenvolvimento motor ocorreu em sete pacientes. O quadro motor tem sido, em geral, estável ou lentamente progressivo. Houve comprometimento difuso da musculatura esquelética, incluindo-se aquela inervada pelos nervos cranianos, associado à hipotonia muscular, arreflexia tendinosa e atrofia muscular. $O$ aspecto facial foi caracteristicamente miopático. Alterações osteoesqueléticas tais como cifoescoliose, lordose, retrações e palato profundo foram freqüentes. Insuficiência respiratória restritiva ocorreu em cinco dos sete pacientes que foram avaliados com prova ventilatória. A dosagem da enzima
CK foi discretamente aumentada somente em um caso. O exame eletroneuromiográfico revelou um padrão miopático em nove pacientes; em três deles, associou-se comprometimento neurogênico e, em outro, descargas miotônicas. Dentre os achados histológicos, destacaram-se o aumento da centralização nuclear na fibra muscular, de 25 a $90 \%$, predominância de fibras do tipo I, variabilidade entre o diâmetro das fibras musculares, alterações da arquitetura interna das fibras musculares e áreas focais de desorganização dos miofilamentos. O prognóstico foi mais reservado na paciente com envolvimento cardiopulmonar, e o comprometimento muscular maior naquele com intensa proliferação conjuntiva, na biopsia muscular.

- Tese apresentada à Universidade Federal de São Paulo - Escola Paulista de Medicina, para obtenção do Título de Mestre em Neurologia. Sāo Paulo 1996. Esta tese foi desenvolvida na Disciplina de Neurologia Clinica do Departamento de Neurologia e Neurocirurgia, da Universidade Federal de São Paulo (UNIFESP) - Escola Paulista de Medicina (EPM), durante o Curso de Pós-Graduaçăo em Neurologia/Neurociéncia, sob orientação do Prof. Dr. Acary Souza Bulle Oliveira e co-orientaçăo do Prof. Dr. Beny Schmidt. O autor foi bolsista do CNPq. 